\title{
Perspectives internationales
}

\author{
Par Ralph W. Roberts, R.P.F., ing. f.
}

Le présent article intègre les contributions de neuf récipiendaires du Prix de foresterie international de l'Institut forestier du Canada. Le hasard a voulu que chacun d'eux s'intéresse et soit rompu à un domaine d'activité distinct. La perspective internationale est leur dénominateur commun. Les points de vue réunis de ces neuf personnes forment une mosaïque fascinante et font voir la scène internationale au travers d'une gamme d'initiatives liées aux forêts. Les sujets traités sont les suivants :

- Coopération internationale en matière de développement

- Recherche forestière

- Semences d'arbres forestiers

- Dialogue international sur les forêts

- Rajustements économiques internationaux

- Enseignement forestier

- Gestion des feux de végétation

- Politique, gestion et leadership

- Conservation et déboisement de la forêt tropicale

Les différentes perspectives sont présentées par ordre chronologique d'attribution du prix aux auteurs.

J'aimerais remercier de son aide Richard Baerg, ing. f., qui a su faire d'une idée d'article une réalité.

\section{8 - Ralph W. Roberts, ing. f.}

Chefforestier

Agence canadienne de développement international

Hull (Québec), Canada

\section{COOPÉRATION INTERNATIONALE EN MATIERE DE DÉVELOPPEMENT}

\section{Le problème}

Par définition, l'aménagement forestier durable ne devrait pas requérir d'aide de l'extérieur. Il devrait s'autofinancer. Il devrait y avoir eu internalisation (des effets externes). Malheureusement, les pratiques forestières sous les tropiques sont loin d'être durables. L'aménagement des forêts pour la seule production de matière ligneuse n'est

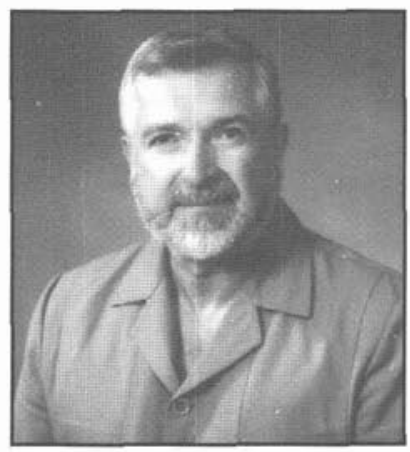
adéquat que sur moins de $5 \%$ de la superficie boisée. La situation est encore pire dans le cas de la multitude d'autres valeurs non ligneuses de la forêt. La demande locale et internationale de bois tropical et de services environnementaux n'a jamais été aussi forte et ne cesse de s'accentuer. Résultat, le Sud a de plus en plus besoin de l'aide du Nord pour parvenir à mieux aménager la forêt tropicale.

Depuis des siècles, les ressources forestières mondiales fluctuent constamment. Cependant, au cours de la deuxième moitié du $\mathrm{XX}^{\mathrm{e}}$ siècle, la dégradation des forêts et la déforestation ont beaucoup diminué. La dégradation des forêts ou un changement du type forestier est un phénomène commun aux deux hémisphères, tout comme la rareté des renseignements à ce sujet. La déforestation, ou évolution de l'utilisation du territoire, est mieux connue. Selon la FAO, le couvert forestier de la planète a régressé de plus de 400 millions d'hectares au cours du présent siècle et, de nos jours, les forêts ne couvrent plus qu'environ 3,4 milliards d'hectares. La forêt tropicale représente quant à elle quelque 2 milliards d'hectares. À l'heure actuelle, la forêt tropicale naturelle disparaît à un rythme d'environ $0,8 \%$ (14 millions d'hectares) chaque année. Dans certains pays, l'établissement de plantations est à la hausse. À l'opposé, la superficie de la forêt tempérée naturelle dans les pays industrialisés n'a augmenté que de $0,1 \%$ au cours des années 1980 , un faible taux de croissance qui se maintient toujours.

\section{L'aide canadienne}

Le Canada a amorcé une coopération internationale en foresterie peu après la Deuxième Guerre mondiale dans le cadre du Plan de Colombo. À cette époque, l'aide était axée sur le développement de l'industrie forestière. L'énoncé de politique étrangère intitulé « Le Canada dans le monde » (février 1995) souligne que la foresterie est un secteur important pour le Canada et pour les pays en développement avec lesquels il collabore par l'entremise de son Programme d'aide publique au développement (APD). Il apporte un changement important en ce qu'il met davantage l'accent sur les aspects sociaux et culturels de l'aménagement forestier. Il vise toujours à continuer de soulager la pauvreté, mais fait jouer aux collectivités rurales un rôle plus grand dans la gérance des forêts et dans la manière dont celles-ci sont aménagées. À l'heure actuelle, une aide annuelle d'environ 50 millions de dollars est accordée à quelque 120 projets menés dans plus de 50 pays. Cette somme comprend les contributions versées à des activités forestières des Nations Unies et d'autres organisations internationales. Elle représente environ $2 \%$ de l'APD du Canada, pourcentage à peu près semblable à celui consacré à la foresterie par l'ensemble des pays. Pour atteindre l'objectif d'aide au développement internationalement accepté qui s'élève à $0,7 \%$ du PNB, il faudrait plus que doubler en proportion notre aide à la foresterie et à la conservation.

\section{Transfert de technologie}

Au cours du dernier millénaire, la technologie a évolué à un rythme sans précédent, surtout dans les pays industrialisés du Nord. Toutefois, les pays en développement n'y ont pas souvent accès. Ceux qui ont un couvert forestier peu étendu ont généralement le besoin le plus criant de systèmes de soutien qui permettent le transfert de ces technologies ainsi que le renforcement des capacités et la mise en valeur des ressources humaines. À ce titre, ils constituent un groupe ciblé par les organismes d'aide au développement. Le transfert de technologies respectueuses de l'environnement dans le secteur forestier sera un volet important des stratégies internationales élaborées pour permettre à ces pays de gérer, de conserver et d'aménager durablement leurs forêts 


\section{Aide financière}

L'aide financière à la mise en valeur de la forêt tropicale évolue aussi rapidement. Fait paradoxal, les activités forestières appuyées par l'APD du secteur public ont énormément diminué depuis le Sommet de Rio de 1992. D'autre part, l'aide financière correspondante du secteur privé a beaucoup augmenté, une tendance qui devrait se poursuivre. Outre ces deux grandes sources de financement, des instances internationales débattent actuellement de la création d'un Fonds mondial pour les forêts. Un tel mécanisme favoriserait et appuierait l'intégration d'activités forestières à des projets de gestion des ressources naturelles de plus grande portée. Toutefois, bien que le but puisse être similaire, les objectifs visés par les parties intéressées des secteurs public et privé et du Fonds mondial pour les forêts seront tous quelque peu différents. Par conséquent, la création d'une agence de développement et de promotion des investissements, un nouvel organisme international qui agirait comme courtier et faciliterait les flux financiers, le transfert de technologie et le renforcement des capacités, semble augurer que les objectifs variés des investisseurs seront pris en compte de la manière la plus efficace possible.

\section{Programmes forestiers nationaux}

Le Programme d'action forestier tropical du milieu des années 1980 a évolué et a jeté les bases de nombreux programmes forestiers nationaux (PFN) dans les pays en voie de développement. Ces PFN définiront de plus en plus les priorités que fixeront les gouvernements pour le secteur forestier. Il a été sans cesse démontré que la volonté politique d'élaborer, de mettre en oeuvre et d'orienter les politiques forestières est l'élément clé d'une gestion efficace des ressources forestières nationales. Les pays qui manifestent une ferme volonté politique en mettant en oeuvre des PFN peuvent s'attendre à obtenir une part importante des flux de capitaux privés et publics. De plus, les PFN continueront d'être un outil important à l'appui de la coordination des efforts entre les bailleurs de fonds nationaux et internationaux et le secteur privé.

\section{Leadership}

Le leadership et la gestion publique à l'échelle internationale sont des questions qui ont été largement débattues au cours des deux dernières décennies. À cet égard, une convention internationale sur les forêts pourrait procurer d'importants avantages. Toutefois, la plupart des pays en développement ne l'avaliseront que lorsqu'ils seront assurés de l'aide financière et du transfert des technologies nécessaires pour les aider à remplir leurs obligations en vertu d'un instrument juridiquement contraignant. Un autre moyen efficace de renforcer le leadership international serait de créer, comme l'a proposé la Commission mondiale des forêts et du développement durable, un conseil de sécurité des forêts composé des pays qui se classent parmi les premiers en termes de couvert forestier ainsi que de production, de commerce et de consommation de produits du bois.

\section{Perspectives d'avenir}

Les questions que nous aurons à résoudre sont nombreuses et complexes et nous devrons nous y attaquer avec plus de vigueur que jamais si nous voulons assurer l'avenir des forêts de la planète au-delà du prochain millénaire. L'APD et le Canada continueront d'avoir un rôle à jouer. Nous sommes un pays riche en ressources et en compétences forestières : nous nous devons d'agir. Les consultants, les organismes publics, les écoles forestières, les entreprises industrielles et les organismes non gouvernementaux ont tous un rôle à jouer. Nous avons beaucoup à offrir et aussi beaucoup à gagner.

\section{0 - James H. Cayford, R.P.F. Consultant forestier, Nepean (Ontario)}

\section{LA RECHERCHE FORESTIERE}

La recherche forestière a toujours été du ressort des gouvernements nationaux et financée en fonction de l'ordre de priorité national. Même si certains pays, en particulier d'Europe, d'Amérique du Nord et d'Asie, ont mis en oeuvre d'importants programmes à cet égard, la plupart des pays de la planète ont fait peu de cas de la

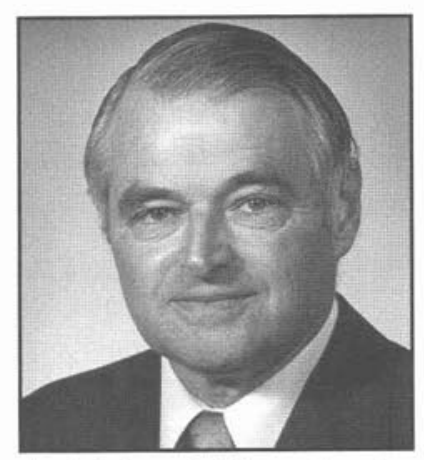
recherche forestière. La faible priorité donnée à la recherche forestière est souvent attribuable au fait que de nombreux gouvernements intègrent les forêts à l'agriculture et accordent souvent une priorité plus faible à la foresterie.

Malgré le rôle de l'État dans la recherche forestière, des organismes de recherche forestière de l'Allemagne, de la Suisse, de la France et de l'Autriche ont mis sur pied en 1892 une union internationale pour faciliter la coopération dans ce domaine. Au début, celle-ci était résolument tournée vers l'Europe. Toutefois, en 1929, elle s'est donné une vocation véritablement internationale et a changé son nom pour celui d'Union internationale des instituts de recherches forestières (IUFRO). Cet organisme, ardent promoteur de la collaboration entre les chercheurs, s'emploie actuellement à jouer un plus grand rôle dans la promotion du dialogue entre les scientifiques et les décideurs et dans la compilation et la synthèse de la documentation disponible à l'échelle mondiale. Le Canada a joué un rôle important dans l'IUFRO. Il a convoqué de nombreuses réunions de l'IUFRO, des Canadiens ont occupé des postes de haut niveau au sein de l'organisation, notamment de vice-président à deux reprises, et le $\mathrm{XIX}^{\mathrm{e}}$ Congrès forestier mondial de l'IUFRO s'est tenu à Montréal.

Lors de son congrès mondial de 1981, au Japon, l'IUFRO a été mise au défi de renforcer la recherche sur les ressources forestières dans les pays en développement. Par la suite, soit en 1993, elle a mis sur pied un programme spécial pour les pays en développement. Ce programme permanent visait à définir les besoins et les priorités en matière de recherche, à apporter de l'aide et à donner une formation aux scientifiques des pays en développement ainsi qu'à offrir des ateliers de formation.

En 1977, le Conseil (maintenant appelé le Centre) international pour la recherche en agroforesterie voyait le jour avec l'appui de l'Agence canadienne de développement international (ACDI) et du Centre de recherches pour le développement international (CRDI). Le siège de cet organisme se trouve maintenant à Nairobi, au Kenya. Plus récemment, soit en 1993, on mettait sur pied à Bogor, en Indonésie, le Centre pour la recherche forestière internationale (CIFOR) avec l'appui de la plupart des pays industrialisés. Ces deux centres jouent des rôles de premier plan dans la recherche forestière et agroforestière de portée internationale. 


\section{Enjeux, préoccupations et considérations internationales \\ Information}

L'un des grands enjeux concerne la nécessité de résumer et de synthétiser la masse de renseignements actuellement disponibles, mais qui n'est pas sous une forme compréhensible pour les décideurs, voire pour d'autres scientifiques. L'IUFRO a créé un groupe de travail sur un service mondial d'information forestière chargé de mettre sur pied une métabase de données et des bibliothèques virtuelles qui puissent servir de centre documentaire de première ligne sur des questions forestières.

\section{Politique forestière}

Les scientifiques ont souvent fixé l'ordre de priorité des recherches sans véritablement tenir compte des besoins des décideurs. Même si les chercheurs n'élaborent pas de politiques, ils doivent être en mesure de conseiller les décideurs sur les impacts éventuels des décisions stratégiques. À l'issue d'un examen externe récent, il a été recommandé que l'IUFRO améliore l'interface entre la science et la politique forestière.

\section{Collaboration intersectorielle et interdisciplinaire}

Pour être vraiment efficaces, les chercheurs en foresterie appartenant à différentes disciplines doivent travailler main dans la main, sans craindre d'être désavantagés sur le plan concurrentiel ou d'être incapables de publier ou de ne pas pouvoir être admis au sein d'équipes interdisciplinaires. De plus, la recherche forestière doit tenir compte non seulement d'écosystèmes forestiers particuliers, mais également des grandes questions liées à la gestion et à l'utilisation du territoire. Une telle recherche intersectorielle et interdisciplinaire exigera que les établissements de recherche et les chercheurs eux-mêmes modifient certaines de leurs façons de faire et reconnaît la nécessité d'une collaboration entre les spécialistes des sciences biophysiques et socioéconomiques.

\section{Thèmes scientifiques clés}

$\mathrm{Au}$ cours de la prochaine décennie, certains thèmes seront prioritaires, notamment :

- les forêts et l'évolution de l'environnement et du climat;

- la conservation et l'amélioration des ressources génétiques forestières;

- l'aménagement forestier durable ainsi que son évaluation et son suivi grâce à l'élaboration de critères et d'indicateurs;

- l'évaluation des produits et des services tirés des forêts;

- la mise au point de produits et de procédés nouveaux;

- l'évaluation des risques et des bienfaits rattachés aux arbres génétiquement modifiés.

\section{Le dialogue international}

Les participants à la Conférence des Nations Unies sur l'environnement et le développement (CNUED) de 1992 ont clairement reconnu que les forêts de la planète sont dans une situation critique et que la communauté internationale doit de toute urgence déployer des efforts coordonnés pour s'engager dans la voie de la gestion durable des ressources forestières. La recherche forestière a été expressément désignée comme l'un des domaines dont il faut s'occuper.

Dans la foulée de la CNUED, la Commission du développement durable (CDD) des Nations Unies a mis sur pied un Groupe intergouvernemental sur les forêts (GIF) auquel a succédé un
Forum intergouvernemental sur les forêts (FIF). La recherche forestière a été l'un des thèmes étudiés par le GIF et le FIF qui ont très bien réussi à cerner les besoins en matière de recherche à l'échelle internationale. Il faut notamment :

1. renforcer la recherche forestière afin d'informer les décideurs, de résoudre les problèmes nationaux et de respecter les priorités nationales;

2. encourager les pays à accorder la priorité au secteur forestier en affectant des ressources au renforcement des capacités nationales en matière de recherche;

3. accroître les ressources consacrées à la recherche et faire contribuer les organisations internationales et les institutions financières au financement de la recherche dans les pays en développement;

4. utiliser convenablement le savoir traditionnel dans l'aménagement durable des forêts;

5. améliorer la coordination de la recherche et cerner les besoins en matière de recherche interdisciplinaire à l'échelle de la planète et des écorégions;

6. améliorer les mécanismes de recherche pour appuyer l'aménagement durable et la conservation des forêts.

La quatrième et dernière session du FIF se tiendra en février 2000. À l'issue de cette session, le FIF présentera à la Commission du développement durable des Nations Unies un rapport contenant ses recommandations sur chacun des différents thèmes. Les décisions qui seront prises à l'égard de ces recommandations auront un effet déterminant sur le rôle futur de la recherche forestière à l'échelle internationale.

L'auteur désire remercier de leurs commentaires pertinents J. Burley et H. Schmutzenhofer, de l'IUFRO, ainsi que V. Nordin, du Forestry Chronicle.

\section{1 - Ben S.P. Wang \\ Chercheur émérite, Forêt expérimentale de Petawawa, Service canadien des forêts, Chalk River (Ontario)}

\section{LES SEMENCES D'ARBRES FORESTIERS}

Malgré les progrès accomplis récemment dans le domaine de la micropropagation et de la culture in vitro, les semences d'arbres forestiers ont toujours été la principale source de matériel de reproduction utilisé dans les programmes de reboisement. Depuis la fin des années 1970, les activités de recherche et développement liées à sémi-

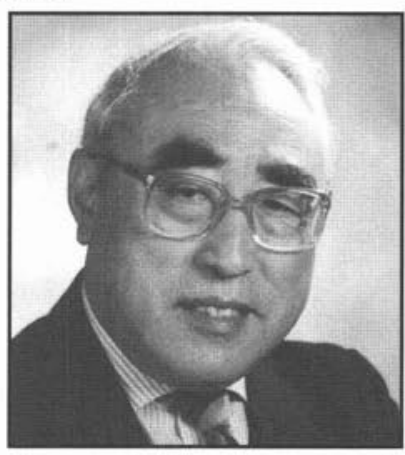
nologie (science des semences) et à ses techniques se sont multipliées parallèlement à l'expansion rapide des programmes de régénération artificielle au Canada. Par conséquent, une foule de renseignements utiles sur divers aspects scientifiques et technologiques de la production des semences a été compilée et mise à la disposition des utilisateurs de semences. Une bibliographie annotée sur l'obtention et le traitement des semences de conifères axée plus particulièrement sur la Colombie-Britannique a été publiée en 1975. Elle recensait plus de 476 articles parus de 1935 à 1974 et traitant des semences forestières 
en général, de leur production, de leur récolte, de leur traitement et d'aspects scientifiques et technologiques. Plus de la moitié de ces articles ont paru pendant les années 1960.

Plus récemment, la recherche en génétique a révélé l'importance de la diversité génétique et de sa conservation grâce à un aménagement adéquat des futurs peuplements de seconde venue. Au Canada, les programmes de reboisement ont commencé à utiliser des semences génétiquement modifiées issues de vergers à graines. Pour tirer le meilleur parti possible de ce matériel de reproduction rare, on a eu recours à des techniques et à de l'équipement améliorés de traitement des semences pour obtenir des graines de qualité qui peuvent être semées à raison d'une seule graine par cavité lors de la production de semis en récipient. Une telle pratique est judicieuse sur le plan génétique, car elle empêche toute disparition possible de familles et les modifications de la diversité génétique résultant des différences d'énergie germinative dues à l'ensemencement de nombreuses semences provenant de lots homogénéisés dans chaque cavité qu'il faut ensuite éclaircir.

En matière de traitement des semences, on a constaté que la stratification froide avait non seulement des effets bénéfiques sur la germination des semences forestières non dormantes (p. ex., de pin gris et d'épinette noire), mais qu'elle améliorait également la manutention, le conditionnement et le vieillissement des graines. On a étudié la vigueur germinative des graines de plusieurs essences forestières et on a découvert que les tests d'accélération du vieillissement et de conductivité s'étaient avérés efficaces et utiles. Dans le domaine de l'entreposage, les progrès en cryopréservation peuvent prolonger la durée de conservation des graines orthodoxes et récalcitrantes; de plus, des recherches plus poussées sur les semences d'essences tropicales ont permis de découvrir l'existence d'un nouveau comportement « intermédiaire » chez les graines entreposées.

À l'échelle internationale, le groupe de recherche sur la physiologie et la technologie des semences de l'IUFRO a beaucoup contribué à l'avancement de la séminologie et de ses techniques. Depuis le début des années 1970, il a sans cesse fait la promotion de la recherche-développement en matière de semences forestières en parrainant à maintes reprises des symposiums scientifiques et techniques internationaux sur divers sujets pertinents dans différents pays. Les comptes rendus publiés ont été bien reçus.

L'Association internationale d'essais de semences (ISTA) a contribué à l'avancement de la séminologie et de ses techniques par l'entremise de son Comité sur les semences d'arbres et d'arbustes (TSSC) et d'autres comités techniques. En plus de mettre périodiquement à jour les méthodes et protocoles d'essai des semences d'arbres qui sont décrits dans les règles internationales établies à partir de résultats des recherches, le TSSC a publié un guide sur les semences d'arbres et d'arbustes (Tree and shrub seed handbook) en 1991 et un autre sur les semences forestières tropicales (Tropical tree seed and shrub handbook) en 1998. La FAO a fait la promotion de la séminologie et de ses techniques grâce à des publications comme le Guide de manipulation des semences forestières et la Conservation ex situ de pollen et de graines, et de cultures in vitro de plantes ligneuses pérennes. De même, le Centre de semences forestières de la DANIDA (Agence danoise de développement international) a publié de nombreux bulletins techniques utiles et a dispensé une formation sur les techniques séminologiques. Le Centre de semences forestières de l'Australie est renommé comme un fournisseur de semences identifiées de nombreuses essences forestières à croissance rapide dans de nombreux pays tropicaux et subtropicaux.

L'Agence canadienne de développement international (ACDI) a aidé l'Asie du Sud-Est à mettre sur pied le projet de centre régional de semences forestières de l'ANASE en Thailande et le projet de centre de semences forestières du SADC au Zimbabwe. L'approvisionnement en semences forestières est l'un des problèmes les plus courants reconnus par la plupart des pays en développement et des bailleurs de fonds internationaux. Ces projets internationaux devraient contribuer à redresser la situation.

Maintenant que le projet de centre régional de semences forestières de l'ANASE de Thailande qu'a appuyé l'ACDI est terminé, il mériterait de continuer à recevoir des fonds pour que les chercheurs et les scientifiques compétents formés par le Canada puissent mener efficacement les activités de recherche-développement qui s'imposent. Ces dernières souffrent d'un manque de financement.

Dans le futur, il faudra porter attention à la recherchedéveloppement dans le domaine de la séminologie et de ses techniques, notamment celle liée aux essences tropicales. Même si les progrès de la culture in vitro semblent prometteurs comme stratégie de rechange, il faudra un certain temps pour parfaire les systèmes de production commerciale de semis. Les aménagistes doivent reconnaître l'importance non seulement de l'origine et de la qualité des semences, mais également de la conservation de la diversité génétique dans les futurs peuplements de seconde venue. Sous les tropiques, la multiplication végétative est une technique bien maîtrisée qui ne nécessite encore que quelques améliorations avant de pouvoir être appliquée à grande échelle.

\section{2 - Jagmohan (Jag) Maini}

Coordonnateur et directeur, Secrétariat du Forum intergouvernemental sur les forêts

\section{DIALOGUE INTERNATIONAL SUR LES FORETS}

Couvrant près du tiers des terres émergées de la planète, les forêts et les « autres terres boisées » (ATB) sont inégalement réparties dans le monde. Bien que pratiquement $60 \%$ des forêts mondiales appartiennent à six pays (la Russie, le Canada, les États-Unis, le Brésil, le Congo et l'Indonésie) et que près de $85 \%$ des forêts et ATB se retrouvent dans environ 25 pays, plus de 70 pays parmi les plus industrialisés appartiennent à la catégorie des pays à faible couvert forestier. $\mathrm{Au}$ cours de la dernière décennie, les forêts sont demeurées une question prioritaire du programme d'action international et sur la scène politique. Le débat international a surtout tourné autour des préoccupations des pays dotés de riches ressources forestières. Toutefois, récemment, les milieux politiques se sont aussi intéressés aux préoccupations propres aux pays en développement à faible couvert forestier, car plusieurs centaines 
de millions de personnes vivent dans ces forêts ou près de cellesci et leur subsistance en dépend.

\section{Les forêts, un enjeu mondial}

Le débat international les forêts auquel participent de nombreuses organisations internationales est complexe et intersectoriel et met en jeu des conventions juridiquement contraignantes et des ententes n'ayant pas force obligatoire. Il a porté sur une vaste gamme de questions liées à la conservation et à l'aménagement durable des forêts et des ATB ainsi qu'au bien-être des personnes qui en dépendent. Bien que le droit souverain des États à mettre leurs ressources au service de leurs priorités nationales et de leurs objectifs stratégiques soit largement reconnu et ne soit pas contesté, un certain nombre de préoccupations ont « internationalisé » la question des forêts, notamment :

- les taux inacceptables de déforestation (13,7 millions d'hectares par année), surtout dans les pays en développement riches en ressources forestières ainsi que la dégradation et les pertes forestières dans les pays à faible couvert forestier causées par les pressions démographiques sans cesse croissantes et leurs impacts sur le bien-être des populations humaines;

- la nécessité de satisfaire à la demande grandissante de certains pays industrialisés pour des « produits écologiques » provenant de « forêts écologiques » certifiées;

- une prise de conscience de plus en plus répandue qu'il faut garder en santé les forêts de la planète en raison de leur valeur écologique et des services qu'elles rendent à l'environnement (p. ex., diversité biologique, séquestration du carbone, conservation des sols et de l'eau);

- la capacité des forêts, dont la superficie ne cesse de diminuer, de répondre à la demande future de produits et services forestiers associée à l'augmentation de la population et à l'essor de l'économie;

- la nécessité de favoriser la coopération entre les pays industrialisés et les pays en développement afin d'assurer la conservation et l'aménagement durable de tous les types de forêt et de remettre en état et de restaurer les forêts et ATB dégradées.

Les participants au débat intergouvernemental qui a eu lieu lors du Sommet de la Terre à Rio (1992) et qui a cours depuis 1995 au sein du Groupe intergouvernemental sur les forêts (GIF) et du Forum intergouvernemental sur les forêts (FIF) ont reconnu que les forêts et les ATB fournissent collectivement un large éventail d'avantages et de services et que leur conservation et leur aménagement durable passent part : i) une harmonisation des politiques intersectorielles, ii) un processus décisionnel participatif auquel doivent contribuer tous les intervenants, iii) un engagement politique à long terme et iv) un aménagement des écosystèmes forestiers basé sur de solides connaissances scientifiques.

Les participants au GIF/FIF ont également souligné que l'élaboration de programmes forestiers nationaux (PFN) est un élément essentiel pour atteindre les objectifs d'aménagement forestier durable et qu'elle devrait procéder d'un processus participatif ouvert, transparent et global ainsi que d'un engagement politique ferme et soutenu. De plus, l'élaboration d'un PFN ne peut être entreprise en vase clos, sans tenir compte du contexte binational, régional et mondial.

\section{Incidences pour le Canada}

Le Canada possède environ $10 \%$ des forêts de la planète et occupe quelque $20 \%$ du marché international des produits forestiers. Sa politique forestière reconnaît depuis longtemps les dimensions binationales (p. ex., avec les États-Unis), régionales (p. ex., dans le cadre de l'ALENA) et mondiale des forêts. Le contenu et la démarche de la Stratégie nationale sur les forêts et l'Accord canadien sur les forêts, dont les premières versions ont été élaborées au milieu des années 1980 à l'aide d'un vaste processus de consultation multilatérale, étaient très avant-gardistes par rapport aux autres pays. Ces documents reconnaissaient que le Canada avait tout intérêt à protéger ses forêts et à les garder en santé, tant pour son propre bien que pour celui de la planète. La politique forestière canadienne devrait être à long terme, prévoir une harmonisation des politiques intersectorielles et, par conséquent, servir d'indicateur de l'engagement du Canada envers le développement durable.

Lors des négociations sur les forêts du Sommet de la Terre, les dirigeants canadiens ont contribué à structurer le débat sur les forêts et à définir les priorités mondiales à cet égard et étaient pleinement conscients que les décisions et les ententes intergouvernementales sur les forêts auraient un impact direct sur la politique forestière du Canada. Le Canada a orienté le débat international sur les forêts et en a subi l'influence.

Dix ans après le sommet de Rio, le Canada doit maintenant faire le point sur sa politique forestière dans le nouveau contexte mondial et réfléchir aux mesures qu'il doit prendre en priorité à l'échelle internationale et nationale.

\section{À l'échelle internationale}

Continuer de jouer un rôle de premier plan sur la scène internationale à titre d' «État forestier » d'importance en défendant les préoccupations des pays à couvert forestier riche ou faible. Les enjeux les plus préoccupants pour les pays forestiers comme le Canada sont d'ordre environnemental, commercial et économique, tandis que ceux des pays en développement à faible couvert forestier concernent le bien-être des populations humaines, enjeux qui sont tous en harmonie avec les objectifs de la politique étrangère du Canada. Les activités suivantes sont proposées :

- Entreprendre une analyse systématique des progrès accomplis à l'échelle internationale afin de cerner les nouvelles possibilités à exploiter liées à l'accroissement prévu de la demande de produits et de services forestiers.

- Accroître considérablement le niveau des ressources affectées à long terme et en permanence à la coopération internationale sur les forêts, en portant une attention particulière aux pays à faible couvert forestier.

- Commercialiser non seulement les produits forestiers du Canada, mais également le savoir-faire canadien sur les forêts, c.-à-d. le traitement des connaissances et le traitement des matériaux.

\section{À l'échelle nationale}

- Accorder aux forêts un statut politique de haut niveau (p. ex., ministériel) allant de pair avec l'importance de cette ressource pour le Canada et permettant au gouvernement fédéral de jouer un rôle prépondérant à l'échelle nationale et internationale.

- Mettre sur pied un Forum sur les forêts de haut niveau afin de permettre aux principaux intervenants d'amorcer un 
dialogue sérieux pour orienter la mise en oeuvre de la Stratégie nationale sur les forêts au Canada et pour définir le rôle et les responsabilités du pays à l'échelle internationale.

- Examiner la formation professionnelle et technique au Canada pour s'assurer que le pays possède les compétences scientifiques, techniques et professionnelles modernes nécessaires pour s'attaquer aux problèmes complexes de l'aménagement des forêts du Canada. Renforcer la recherche sur les écosystèmes forestiers et la politique forestière.

\section{4 - George S. Nagle, Ph.D., R.P.F.}

Président, économiste principal, Nawitka Renewable Resource Consultants Ltd., Sooke (C.-B.)

\section{LES RAJUSTEMENTS ÉCONOMIQUES INTERNATIONAUX ET LE} SECTEUR FORESTIER

David Suzuki est convaincu que l'économie moderne est fondamentalement déconnectée du monde réel et que c'est en fait une illusion. Or, les pays doivent opérer des choix socio-économiques pour aménager des ressources rares de façon durable et gérer leurs différences avec civilité et dans le respect de la justice. Des rajustements économiques

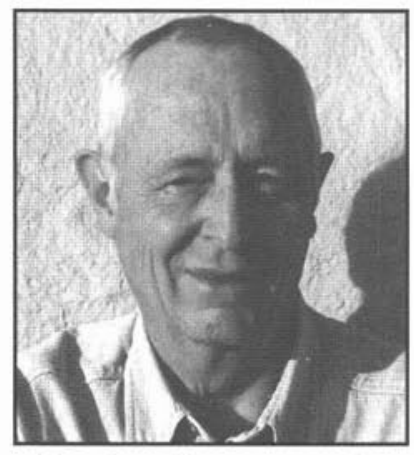
s'imposent donc à l'échelle internationale, et il y en aura. Alors que s'ouvre la conférence de l'OMC à Seattle, nous examinerons brièvement les protagonistes et quelques-uns des enjeux de la nouvelle «mondialisation » des rajustements.

\section{Les protagonistes}

Les pays développés, de concert avec le Groupe de la Banque mondiale, décident dans une large mesure de l'orientation et de l'ampleur du commerce mondial, des mouvements de capitaux et de l'aide publique au développement (APD). Il leur incombe donc de concevoir et de protéger les instruments (devises) des échanges internationaux.

Les pays " émergents » comme la Russie et les pays d'Europe de l'Est, les géants d'Asie en développement et les pays d'Amérique latine et d'Asie du Sud-Est où il s'opère des réformes rapides, revêtent eux aussi de l'importance.

Plusieurs pays pauvres en sont aux premiers stades des réformes et, avec un peu de chance, ils réussiront à obtenir de l'aide et à en faire bon usage. Un autre groupe de pays très pauvres poursuivent leur lutte sans le bénéfice de capitaux, d'un libre échange avec réciprocité, de technologies ou d'une saine gestion des affaires publiques.

Parmi les autres protagonistes importants, il faut citer la famille des organisations non bancaires de l'ONU, un vaste ensemble de multinationales, les marchés monétaires internationaux et les ONG internationales.

\section{Rapports entre l'aide et le commerce international}

En dépit de rajustements assez abrupts dans les pays développés, les ajustements les plus draconiens des échanges mondiaux soient encore à l'étude et ne seront pas amorcés avant le nouveau millénaire. Les pays émergents/en développement doivent avoir plus facilement accès aux vastes marchés de consommation que sont le Japon, l'Europe et l'Amérique du Nord pour exporter leurs produits, de même qu'aux technologies industrielles qu'ils doivent importer. De nombreux obstacles au commerce persistent entre les pays des groupes émergents et en développement.

Les échanges commerciaux entre l'Est et l'Ouest et entre le Nord et le Sud ont souvent été alimentés par les mouvements d'aide publique, les prêts et d'autres transferts de capitaux. De plus en plus de gens pensent que ce rapport n'a pas toujours été sain. L'argent de l'extérieur n'a l'effet désiré que si un pays est doté d'institutions et de politiques économiques solides.

\section{Réforme monétaire}

L'instabilité des devises est souvent le reflet de politiques économiques nationales malavisées. Le besoin d'avoir des liquidités pour stabiliser les devises a été l'un des principaux leviers qu'ont utilisé les banques internationales, l'APD et les milieux économiques régionaux pour tenter d'orienter les politiques nationales.

Seuls les États-nations peuvent maintenir les conditions nécessaires au soutien de leur propre devise. Le Fonds monétaire international et les États qui le composent voudront s'assurer à l'avenir que l'excédent des marchés ou les mesures spéculatives sur les marchés monétaires ne produisent pas d'effets pervers en période de rajustement.

\section{Aménagement structurel}

L'amélioration des structures économiques (marchés, prix, régime de concurrence) et des institutions publiques (démocratie, saine gestion publique) stimule la durabilité du développement socio-économique. Il n'est pas rare que les entreprises d'État mettent à mal l'épargne nationale. Dans bien des pays, l'efficacité et la croissance ont été sérieusement compromises par la corruption.

Certains pays se sont engagés à opérer des réformes, qui se sont cependant révélées peu pratiques compte tenu de leurs institutions et structures économiques. À l'avenir, les politiques de la Banque mondiale et de l'APD tenteront de susciter l'amélioration de la conjoncture pour un aménagement structurel axé sur le marché avant de fixer des objectifs précis pour la réforme des structures.

\section{Incidences sur la foresterie mondiale et la conservation}

Les problèmes de l'environnement planétaire et la majoration des coûts qu'impose une production respectueuse de l'environnement font en sorte qu'aucun pays ne peut ouvrir ses marchés sans tenir compte du régime environnemental des pays expéditeurs. Cela ouvre la voie à une nouvelle pléthore de problèmes de commerce et d'aménagement, notamment au chapitre des produits forestiers.

Le rôle des organismes non gouvernementaux internationaux (ONGI) a pris une telle ampleur que certains vont jusqu'à prévoir que les systèmes internationaux d'adaptation sociale et environnementale pourraient échapper au pouvoir des États.

Un important intervenant à l'échelle mondiale, l'ONU, demeure en grande partie «non ajustée ». En particulier, les institutions responsables des forêts, de la conservation des ressources et de l'environnement souffrent de lacunes persistantes sur les plans de l'analyse stratégique, de la coordination des efforts et du leadership, en dépit des réformes opérées depuis une décennie. 
Certes, il y a matière à amélioration au niveau des techniques, mais les preuves d'effets nuisibles sur les forêts attribuables au rajustement économique ne sont pas convaincantes. De nombreux instruments perfectionnés d'aménagement des forêts tropicales font leur apparition, et on peut encore espérer une réforme de l'ONU. Tout permet de croire que l'aménagement forestier durable ira de pair avec le rajustement économique au nouveau millénaire.

\section{Incidences au Canada}

Le Canada est confronté à ses propres problèmes d'ajustement. L'avantage que l'on attendait de la dévaluation du dollar sur les exportations de bois d'oeuvre a été fortement limité par les mesures compensatoires décrétées par les ÉtatsUnis. Le secteur forestier se heurte à la concurrence internationale farouche des producteurs qui évoluent dans des économies récemment réformées ou restructurées. Le secteur forestier canadien a incontestablement ressenti les pressions qu'exercent les groupes d'ONGI dans divers forums internationaux.

Le rôle des multinationales n'est pas prêt de s'estomper dans le secteur forestier, même si de plus petites entreprises y trouvent elles aussi leur compte. Le principal problème structurel du secteur demeure la propriété publique des ressources ligneuses.

Il serait réconfortant de voir le Canada « prêcher par l'exemple » dans la lutte mondiale pour la durabilité des forêts. L'aide publique au développement consentie par le Canada dans le secteur des forêts baisse régulièrement depuis près de dix ans. Cela cadre mal avec notre rôle de principal exportateur mondial de produits forestiers ou avec nos prises de position sur la conservation de la planète et les conventions sur l'environnement.

\section{6 - John K. Naysmith, R.P.F.}

Doyen fondateur et professeur émérite à la faculté de foresterie de l'Université Lakehead, Directeur, Ghana-Canada IN CONCERT Program Directeur, Nepal Resource Conservation and Community Outreach Program. Thunder Bay (Ontario)

\section{L'ENSEIGNEMENT FORESTIER}

L'Ontario a son Fonds de réserve forestier et l'Écosse, son Borders Community Forest Trust. Ces deux initiatives ont un but commun : la santé des forêts.

Récemment, le gouvernement finlandais a décidé de préserver 77000 hectares de vieille forêt dans le nord de la Finlande. À peu près en même temps, le gouvernement de

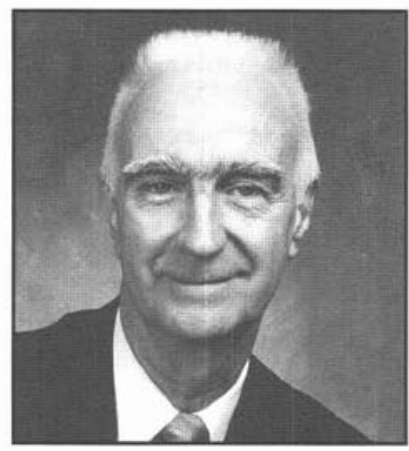
Colombie- Britannique décidait de protéger ses vieilles forêts et prairies alpines dans la vallée de Stein en convertissant une centaine de milliers d'hectares en prairie-parc.

S'agit-il de nouvelles façons d'aménager les terres forestières? Le programme de foresterie communautaire au Népal a abouti jusqu' ici à la constitution de plus de 5000 groupes d'utilisateurs de la forêt. L'une des onze forêts modèles du Canada, la forêt modèle du Bas-Saint-Laurent, dans l'est du Québec, permet aux métayers forestiers d'aménager une forêt et de partager les bénéfices avec son propriétaire.

Les étudiants ont de plus en plus besoin de savoir ce qui se fait dans le reste du monde. Les leçons tirées de l'expérience des autres leur seront bénéfiques lorsqu'ils travailleront avec leurs homologues d'autres pays.

L'enseignement forestier au XXI ${ }^{\mathrm{e}}$ siècle sera une aventure passionnante. Pourquoi, vous demandez-vous? Parce qu'il faut former des forestiers qui possèdent les connaissances et les talents nécessaires pour préserver les forêts du globe avec leurs diverses valeurs, tout en tenant compte du bien-être des habitants de la planète et de leurs divers besoins.

L'une des caractéristiques d'une université idéale est le libre échange d'idées entre professeurs et étudiants. Il serait enrichissant d'élargir la portée et de diversifier la circulation de ces idées pour que les connaissances, les expériences vécues, les résultats des recherches et les exemples pratiques puissent facilement s'échanger entre professeurs et étudiants canadiens et leurs homologues d'autres pays.

Des contacts rapides, directs et « personnels », tels sont quelques-uns des termes qui viennent à l'esprit lorsqu'on songe à la transmission du savoir à grande vitesse par l'Internet, les vidéos par satellite et les téléconférences. La libre circulation des idées prend une tout autre dimension lorsqu'on applique les principes de l'enseignement à distance au contexte mondial. Il s'agit peut-être du mode métadisciplinaire plutôt qu'interdisciplinaire d'aménagement des forêts dont Jack Westoby aimait à parler.

La perspective internationale en matière d'enseignement forestier bénéficie grandement d'initiatives comme le programme Partenariats universitaires en coopération et développement de l'Agence canadienne de développement international. $\mathrm{Ce}$ programme offre d'excellentes occasions aux écoles de foresterie du Canada de participer directement à des projets dans le monde entier.

Les occasions d'apprentissage ne sont pas négligeables pour les professeurs et les étudiants qui participent à des projets de développement international. Au nombre des retombées possibles, mentionnons la possibilité d'intégrer des études de cas et des expériences vécues dans les matériels pédagogiques et les discussions en classe. Sans oublier bien sûr les avantages réciproques qu'il y a à inviter des étudiants et des professeurs de pays partenaires à participer à des programmes dispensés au Canada.

L'éloignement géographique a de moins en moins d'importance et des étudiants de tous les coins du monde ont de plus en plus d'occasions de mieux saisir les enjeux internationaux et de contribuer à relever les défis.

Peter J. Murphy a déjà dit que l'aménagement forestier durable n'est pas tant une destination qu'un voyage que nous devons faire ensemble. C'est un voyage passionnant, à n'en point douter. Et ce qui le rend encore plus passionnant, c'est le nombre croissant de problèmes d'envergure internationale. À l'instar de la plupart des voyages, le plaisir commence au moment de la planification et, pour de nombreux étudiants du $\mathrm{XXI}^{\mathrm{e}}$ siècle, l'enseignement forestier dans une perspective internationale marquera le début du périple. 
1997 - John F. Goodman, R.P.F.

Consultant en feux de forêts, Ancien sous-ministre adjoint et chef de la Direction de la gestion de la lutte contre les incendies, province de l'Ontario, Ottawa

\section{GESTION DES FEUX DE VÉGÉTATION \\ Introduction}

Le feu est un phénomènes naturels les plus variables et les plus dynamiques que l'on connaisse. Il est essentiel à la préservation de nos écosystèmes car il oblige les forêts à se renouveler et à prospérer. Au seuil du nouveau millénaire, les feux de végétation continuent de faire planer une grave menace sur les écosystèmes forestiers, la

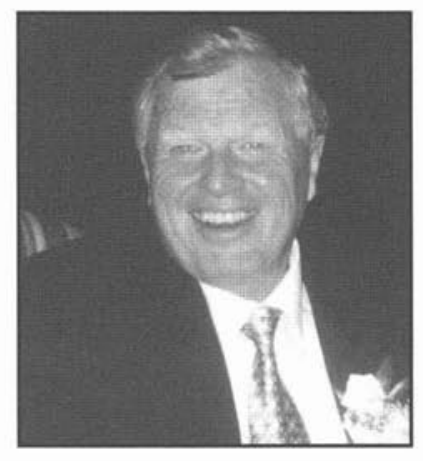
sécurité publique et le bien-être économique des régions du monde vulnérables à ce genre de phénomène.

\section{Le problème}

L'incidence des feux de végétation et les superficies affectées augmentent considérablement depuis quelques années. Certaines estimations portent à croire que jusqu'à un milliard d'hectares de forêt sont dévastés chaque année par le feu. Les feux de végétation peuvent avoir de profondes répercussions sur la durabilité des écosystèmes forestiers aussi bien dans les pays industrialisés que dans les pays en développement. Les régimes d'inflammabilité changent au gré du réchauffement de la planète, des changements climatiques, de l'accumulation de combustibles, de l'explosion démographique et de la pauvreté des politiques sur l'affectation des terres. Dans les pays industrialisés, les politiques fructueuses de lutte contre les incendies fondées sur des technologies de pointe ont amélioré les régimes d'inflammabilité et réduit la fréquence des feux.

En cette fin de siècle, les grands feux sont devenus monnaie courante et ils retiennent l'attention du monde entier. Par exemple, en 1983, en Australie, la catastrophe du Mercredi des Cendres a fait 77 morts, brûlé 340000 hectares et détruit 2500 maisons. En 1987, un vaste incendie dans le nord-est de la Chine a ravagé 1,3 million d'hectares et provoqué de lourdes pertes en vies humaines et en établissements. D'autres incendies de même ampleur ont dévasté certaines parties des États-Unis, du Mexique, de la Russie, du Canada, de l'Asie du Sud-Est et du Brésil. Les feux de végétation se sont multipliés de manière spectaculaire ces dernières années, et d'aucuns prétendent qu'ils ont atteint des proportions critiques.

\section{Les effets}

L'ampleur de l'impact des feux non dirigés varie d'une région à l'autre et dépend de l'importance, de l'intensité et de la fréquence du phénomène. Tout le monde s'accorde à penser que les incidences sont manifestes autant sur les populations humaines que sur l'environnement. Par exemple, un feu non maîtrisé contribue pour beaucoup aux concentrations atmosphériques de dioxyde de carbone, qui font monter la température et finissent par entraîner des changements climatiques. Mais, surtout, ce qui revêt une importance critique, c'est la perte de carbone séquestré et la dégradation générale des écosystèmes, qui perdent de leur diversité et de leur durabilité. Les coûts de la lutte contre les incendies sont énormes et mettent souvent à l'épreuve la capacité de nombreux pays industrialisés et en développement d'absorber les dépenses récurrentes que ce fléau entraîne.

Les enjeux sont les mêmes pour tous les pays vulnérables au feu. Il arrive que l'inadaptation des politiques sur l'affectation des terres et l'absence d'éthique environnementale contribuent à d'importants incendies de végétation. Dans le monde en développement, la conversion des forêts, les transmigrations et la pénurie d'infrastructures et de technologies établies entraînent des pertes inacceptables. Cela a de graves répercussions sur la durabilité des écosystèmes forestiers.

L'évolution est toutefois une caractéristique inhérente des écosystèmes forestiers, et le feu est donc un agent de changement naturel. Le résultat peut être positif ou négatif, selon le point de vue.

À l'échelle internationale, des efforts louables ont été déployés, depuis la planification du paysage jusqu'aux technologies de lutte évoluées en passant par les systèmes informatisés d'aide à la décision et de classement des dangers. Par ailleurs, la mise au point d'instruments d'aide réciproque a permis aux organismes d'échanger leurs ressources à l'échelle mondiale. L'expertise et les systèmes canadiens ont été exportés dans le monde entier grâce aux initiatives de la Banque mondiale et à la participation de l'Agence canadienne de développement international et du secteur privé. Des projets ont ainsi été exécutés en Chine, au Bélarus, en Indonésie, en Croatie, au Mexique et en Nouvelle-Zélande.

\section{Quelques difficultés}

Plusieurs difficultés se poseront aux responsables de la lutte contre les feux de végétation au prochain siècle. Mentionnons entre autres le réchauffement de la planète, l'interface entre les milieux urbains et les milieux sauvages, les transmigrations, la conversion des forêts, les cultures sur brûlis, la réinsertion des feux dans l'environnement, les infrastructures et le financement, les valeurs et les risques, le rôle des bénévoles, le partage des ressources et la durabilité de l'environnement. Ces difficultés méritent une grande attention de la part des gouvernements et des milieux scientifiques.

Les feux de végétation continueront de sévir, quelles que soient les interventions de l'homme. Notre défi consiste à gérer le phénomène et à nous efforcer de trouver l'équilibre avec les politiques sur l'affectation des terres et une éthique environnementale compatible avec les besoins sociaux. Au Canada, les services de lutte contre les feux de végétation sont en bonne posture pour contribuer à relever les défis, surtout dans les pays en développement.

Le philosophe Tyron Edwards a dit : «L'enfer, c'est de connaître la vérité trop tard. » Nous connaissons les enjeux et la situation auxquels seront confrontés les responsables de la lutte contre les feux de végétation au siècle prochain. Le véritable objectif sera d'agir à l'échelon international. 
1998 - T.M. (Mike) Apsey, R.P.F.

Expert-conseil en matière de forêts et de politiques commerciales, ancien président, Council of Forest Industries et ancien sous-ministre des Forêts, Colombie-Britannique, Victoria (Colombie-Britannique)

\section{POLITIQUE, GESTION ET LEADERSHIP \\ «... que faites-vous pour marquer le changement de millénaire? »}

L'imminence du nouveau millénaire suscite tout un battage publicitaire, apparemment pour donner aux gens le sentiment de vivre un moment historique et de pouvoir dire après coup : « ... j’y étais ». En réalité, c'est à peu près la seule chose que les gens pourront revendiquer.

Pour ceux qui participent ou qui s'intéressent à la com-

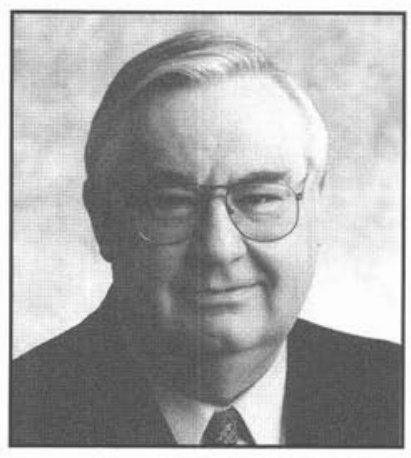
munauté forestière mondiale, cependant, l'arrivée de l'an 2000 peut et doit être un événement marquant. Ce passage marque en effet un tournant décisif dans la longue histoire des rapports que l'humanité entretient avec les forêts, et il coïncide avec le besoin d'entrer résolument dans l'ère nouvelle de l'aménagement des forêts, l'ère de l'aménagement forestier durable. Ce numéro du Forestry Chronicle propose une série d'articles qui rendent bien compte des divers enjeux qui définissent l'état des forêts et de la foresterie d'aujourd'hui. L'heure est venue de faire le point, de sortir de la période qui a consisté à cerner, à étudier et à considérer les différents problèmes et de nous engager avec détermination à définir les visions, les politiques, les institutions, les instruments et les mesures qui nous feront passer à l'ère de l'AFD.

Le fait que nous soyons à un autre tournant décisif ne doit pas nous alarmer ni nous paralyser. Au Canada, lorsque l'ère de la liquidation a cédé la place à l'ère de la conservation il y a un siècle, et que celle-ci a été remplacée par l'ère du rendement soutenu, ceux qui étaient responsables des forêts se sont adaptés et ajustés et ont créé les instruments nécessaires pour bien vivre ces époques. Nous devons en faire autant, et agir dès maintenant.

Tandis que nous nous attelons à la tâche, nous disposons d'avantages que n'avaient pas nos prédécesseurs. Nous avons une connaissance plus approfondie des forêts et de la part qu'elles prennent dans la sécurité de la planète en ce qui a trait au climat, à la qualité de l'air et à l'approvisionnement en eau, de même qu'en ce qui concerne leur contribution économique et sociale à la condition humaine. Nous avons accès à des technologies plus évoluées et à de meilleures voies de communication. En bref, nous sommes en meilleure posture pour nous attaquer à des questions importantes qui aboutiront à l'AFD à l'échelle planétaire.

Qu'est-ce qui nous retient donc sur ce front? Je pense que les progrès sont entravés par quelques facteurs relativement peu nombreux :

- la crainte de ne pas avoir pensé à tous les détails. Nous ne devons pas avoir peur de faire des erreurs. Pour progresser, il faut prendre des risques. La plus grande erreur consiste à ne rien faire;
- l'absence d'instruments de gestion publique internationale efficaces en ce qui concerne les enjeux forestiers mondiaux. De nombreuses initiatives de grande valeur ont été prises dans diverses régions du monde, mais il n'existe pas de système international de gestion publique qui favorise leur cohésion, leur synergie et leur focalisation;

- l'absence du leadership permettant de s'attaquer aux questions forestières critiques. Un leadership efficace et soutenu n'est certes pas une denrée abondante, et le secteur forestier ne fait pas exception à cet égard. Il est impérieux que les hauts dirigeants politiques s'emploient à assurer une gestion efficace de la ressource. Et il en va de même pour l'industrie et les autres intervenants du secteur forestier.

À l'ère de l'aménagement forestier durable pourrait bien se greffer l'ère de l'intégration des intérêts. L'obligation de respecter les impératifs opérationnels de l'AFD finira par échoir à l'industrie. D'autres intérêts veilleront à ce que les régimes à adopter tiennent compte du plus grand nombre de valeurs possible, dont les valeurs économiques, sociales et environnementales.

Le Canada est on ne peut mieux placé pour devenir un chef de file mondial au chapitre des forêts. Il est reconnu comme une puissance dans ce domaine et, à ce titre, il ne doit pas se dérober à ses responsabilités quant aux décisions importantes qui ont trait à l'aménagement forestier durable à l'échelle planétaire.

Il y a des millénaires que les forêts subviennent aux besoins de l'humanité. À la veille du nouveau millénaire, il est grand temps que l'être humain subvienne aux besoins des forêts de la planète. Ceux qui acceptent ce défi pourront dire fièrement qu'ils n'étaient pas seulement des spectateurs au tournant du siècle. Ils auront en effet contribué à l'avènement de l'ère suivante dans l'évolution ininterrompue de l'aménagement des forêts. Et, ce faisant, ils auront permis que l'extraordinaire patrimoine forestier continue de profiter encore à la planète entière au seuil du quatrième millénaire.

\section{9 - John Roper}

Expert-conseil en conservation des forêts, Burnaby (Colombie-Britannique)

\section{CONSERVATION ET DÉBOISEMENT DE LA FORET TROPICALE \\ Une ressource d'importance mondiale}

Au seuil du $\mathrm{XXI}^{\mathrm{e}}$ siècle, la région tropicale compte environ deux milliards d'hectares de forêt. Ces forêts représentent une ressource très précieuse en raison des divers produits économiques et services environnementaux qu'elles procurent. La forêt tropicale est l'habitat de $70 \%$ des espèces végétales et animales de la planète, soit plus

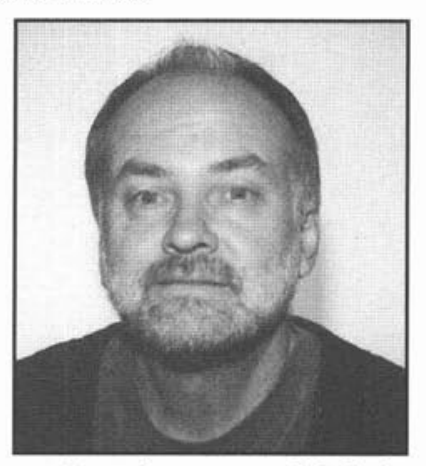
de 13 millions d'espèces différentes. Le volume annuel de bois récolté en forêt tropicale, principalement sous forme de bois de feu, est de plus de un milliard de mètres cubes. Outre les produits ligneux, cette forêt nous fournit un large éventail de produits forestiers non ligneux, désignés sous le nom de « menus » produits forestiers, qui constituent souvent des pro- 
duits forestiers « importants » pour les peuples qui y vivent. Au nombre de ces produits figurent les fibres, les résines, les latex, les fruits et les plantes médicinales traditionnelles. La forêt est souvent une source importante de produits alimentaires, notamment en période de sécheresse et de famine, lorsque les récoltes sont déficitaires. Quelque 500 millions de personnes habitent en forêt tropicale ou en lisière de celle-ci, y compris les 150 millions d'Autochtones dont le mode de vie en dépend.

\section{Déboisement de la forêt tropicale}

Bien qu'on ne connaisse pas avec exactitude la superficie forestière initiale, on estime que la planète a vu disparaître au cours des 8000 dernières années environ $40 \%$ des six milliards d'hectares de forêt présents à l'origine. La majeure partie des ces pertes est directement imputable à l'activité humaine au cours du XX $\mathrm{X}^{\mathrm{e}}$ siècle. La déforestation s'entend de la disparition permanente de forêts au profit d'autres utilisations du territoire et est maintenant largement reconnue comme l'un des problèmes environnementaux les plus graves de notre temps qui se double de lourdes conséquences économiques et sociales à long terme. À l'heure actuelle, 14 à 16 millions d'hectares de forêt tropicale sont convertis chaque année à d'autres utilisations, principalement agricoles.

\section{Causes et agents}

Au nombre des facteurs favorisant la déforestation figurent la pauvreté, la cupidité, la soif de pouvoir, la croissance démographique et l'analphabétisme. Les causes indirectes du fléau sont des politiques gouvernementales inopportunes, la rareté des terres, les forces du marché national et mondial, la sous-évaluation des forêts naturelles, la faiblesse des institutions gouvernementales et les facteurs sociaux. Les causes directes plus visibles de la déforestation sont les utilisations des terres qui concurrencent les forêts naturelles (comme l'agriculture, l'élevage, la mise en place d'infrastructures et l'exploration minière et pétrolière). L'exploitation forestière, la récolte du bois de feu et la création de nouvelles plantations jouent aussi un rôle dans le phénomène de déforestation. Parmi les principaux agents de déforestation, les personnes qui font disparaître les forêts, figurent celles qui s'adonnent à la culture itinérante sur brûlis, à l'agriculture commerciale, à l'élevage, à la coupe du bois, à la récolte du bois de feu, à la mise en place d'infrastructures et à d'autres activités.

\section{Peut-on sauver la forêt tropicale?}

Même s'il est impossible de mettre complètement fin à la déforestation dans un avenir prévisible, il existe de nombreux moyens de l'enrayer et de réduire ses impacts négatifs :
1, protection et aménagement des forêts restantes - soutien accru des réseaux de zones protégées représentatifs d'écosystèmes forestiers importants - adoption de pratiques conjointes d'aménagement des forêts et/ou d'approches de forêt modèle qui favorisent la participation d'une vaste gamme d'intervenants - amélioration de la qualité et de la fiabilité de l'information sur les ressources forestières - et adoption à grande échelle de pratiques véritablement durables d'exploitation forestière;

2, développement socio-économique des régions rurales amélioration de la productivité des utilisations concurrentes des terres, à savoir l'agriculture de subsistance et l'élevage extensif, pour contribuer à réduire les pressions en vue de la conversion d'autres terres boisées - promotion de l'agroforesterie en vue d'accroître la production agricole et de reboiser les terres dénudées - augmentation de la superficie des plantations établies à l'aide d'espèces appropriées - restauration des terres dégradées, notamment dans les bassins versants fragiles;

3 , réformes des politiques et des institutions - accroissement de la participation de tous les intervenants au processus décisionnel et au partage des bénéfices - augmentation des investissements dans la recherche forestière, notamment la recherche sur la politique, l'utilisation du territoire, les systèmes agroforestiers, les systèmes sylvicoles, l'amélioration des arbres, la gestion communautaire des forêts, l'évaluation forestière, notamment de la valeur des produits et services non marchands, et le savoir traditionnel rattaché aux produits forestiers et aux pratiques d'aménagement réforme des politiques et des institutions gouvernementales qui ont un impact négatif sur la pérennité des ressources forestières - appui de l'enseignement et du renforcement des capacités liés aux enjeux du secteur forestier et appui des programmes forestiers nationaux (PFN) comme le moyen optimal de réaliser un large consensus multisectoriel sur le développement du secteur forestier.

Aucun autre enjeu forestier n'est aussi important pour le bienêtre à long terme de notre planète que la conservation et le déboisement de la forêt tropicale. Même si nous ne connaissons pas toutes les réponses, nous disposons de nombreuses technologies qui peuvent donner des résultats probants, tout comme des méthodes nécessaires pour les mettre en application. Il est temps pour tous les peuples de renouveler leur engagement à vivre en harmonie avec la forêt tropicale avant qu' elle ne disparaisse à tout jamais. 